\title{
SPICE TOOLS SUPPORTING PLANETARY REMOTE SENSING
}

\author{
C. Acton ${ }^{a^{*}}$, N. Bachman ${ }^{a}$, B. Semenov ${ }^{a}$, E. Wright ${ }^{a}$ \\ ${ }^{\text {a }}$ Caltech/Jet Propulsion Laboratory 4800 Oak Grove Dr., Pasadena CA 91109 \\ (charles.acton, eric.ferguson, nathaniel.bachman, boris.semenov, edward.wright)@jpl.nasa.gov
}

Commission IV, WG IV/8

KEY WORDS: Observation geometry

\begin{abstract}
:
NASA's "SPICE"* ancillary information system has gradually become the de facto international standard for providing scientists the fundamental observation geometry needed to perform photogrammetry, map making and other kinds of planetary science data analysis. SPICE provides position and orientation ephemerides of both the robotic spacecraft and the target body; target body size and shape data; instrument mounting alignment and field-of-view geometry; reference frame specifications; and underlying time system conversions.
\end{abstract}

SPICE comprises not only data, but also a large suite of software, known as the SPICE Toolkit, used to access those data and subsequently compute derived quantities-items such as instrument viewing latitude/longitude, lighting angles, altitude, etc.

In existence since the days of the Magellan mission to Venus, the SPICE system has continuously grown to better meet the needs of scientists and engineers. For example, originally the SPICE Toolkit was offered only in Fortran 77, but is now available in C, IDL, MATLAB, and Java Native Interface. SPICE calculations were originally available only using APIs (subroutines), but can now be executed using a client-server interface to a geometry engine. Originally SPICE "products" were only available in numeric form, but now SPICE data visualization is also available.

The SPICE components are free of cost, license and export restrictions. Substantial tutorials and programming lessons help new users learn to employ SPICE calculations in their own programs. The SPICE system is implemented and maintained by the Navigation and Ancillary Information Facility (NAIF)-a component of NASA's Planetary Data System (PDS).

This poster highlights additions to SPICE that could be of interest to attendees to the ISPRS symposium.

* Spacecraft, Planet, Instrument, Camera-matrix, Events http://naif.jpl.nasa.gov/naif/aboutspice.html

\section{INTRODUCTION}

The SPICE system came into existence in the early 1990's at the request of NASA scientists who wanted better means to calculate observation geometry associated with their planetary science instruments. They equally wanted a better means to archive and subsequently re-use these so-called "ancillary data" to provide better opportunities for improving science data analyses long after a mission was completed.

SPICE places much of the burden of doing geometry calculations on the shoulders of instrument teams and research scientists, but in so doing it provides this community with a great deal more capability and flexibility than had previously been available (Acton 1996a). Mission control engineers and some scientists produce rather low-level ancillary data, called "kernels," from which the planetary science community can compute a wide assortment of derived quantities such as altitude, LAT/LON, and lighting angles. These computations are facilitated by the existence of a large collection of allied software known as the SPICE Toolkit. A SPICE user integrates a few Toolkit modules (subroutines, APIs) into his/her own application program in order to read data from the SPICE kernels and subsequently compute derived geometry parameters.

While originally focused solely on science data analysis and archive preparation, in the years since its first use on the Mars Observer and Galileo missions it has found a niche in every phase of a mission-from pre Phase A through long after a mission is over. At the same time the use of SPICE has extended beyond the science community, now well supporting mission engineering and space agency infrastructure such as NASA's Deep Space Network.

\section{SPICE TOOLS}

The primary "tool" of the SPICE system is the large library of modules within the SPICE Toolkit. Initiated about 25 years ago, it has continuously grown in capability to meet the everygrowing needs of the space science community.

\footnotetext{
${ }^{*}$ Corresponding author
} 


\subsection{Key Toolkit Attributes}

Given the SPICE system's longevity, a key characteristic of this "tool" is its $100 \%$ backwards compatibility: the NAIF Team never removes or changes a module provided in an official Toolkit release. Of equal importance are the Toolkit's testing and documentation attributes. Each module has an associated test script that is exercised on all $40+$ Toolkit environments supported by NAIF. Thus SPICE users rarely encounter a bug. Each module contains an extensive source code "header" providing comprehensive instructions for its use, and usually also containing one or more working code examples. A consistent documentation style is used throughout the module library making it rather easy for users to quickly grasp what information will be found for every module.

\subsection{New Toolkit Capabilities}

2.2.1 Geometry Finder Subsystem For many years the toolkit allowed for just the computation of some sort of observation geometry parameter-say altitude or instrument boresight surface intercept location-at a user specified time T. Recently, in response to user requests, the inverse process has slowly been added, what NAIF calls the "geometry finder" subsystem (GF). Using any of the family of GF APIs one can use SPICE to find, within an initial confinement window, all the time intervals when a selected geometric condition is TRUE, occultation being an example. Or, one can find all the time intervals when a selected geometric parameter is within a specified range, or has reached a local or global MIN or MAX. Orbital event finding computations are certainly not unique to SPICE. But some implementations make use of mission heuristics and as such do not port well to new orbital conditions, whereas the SPICE implementations are thought to work well under any geometric conditions. This includes consideration of computational performance and accuracy.

The Geometry Finder subsystem capability available in the current Toolkit offers considerable capability, but still more GF computations will be added in future Toolkit releases. A tutorial about the Geometry Finder subsystem is available here: http://naif.jpl.nasa.gov/pub/naif/toolkit_docs/Tutorials/pdf/indiv idual_docs/29_geometry_finder.pdf.

2.2.2 Digital Shape Kernel Subsystem Until very recently all solar system bodies were modelled within SPICE as tri-axial ellipsoids (or sometimes just oblate spheroids). Modern planetary exploration missions have clearly shown such simplistic models to be insufficient. Whether it is the chaotic terrain of comet Churyumov-Gershmenko, or the substantial relief of nearly any large planet or satellite, "smooth" surfaces do not suffice.

To address this lack of fidelity NAIF undertook development of what it calls the Digital Shape Kernel (DSK) subsystem. DSK comprises both a new kind of data file (a new "kernel") and associated Toolkit APIs. DSK is actually like two new data types rolled into one flexible file structure. The first type contains tessellated plate data, best suited for modelling the highly irregular shapes of small bodies such as comet Churyumov-Gerasimenko (Fig. 1) or Phobos. The second type contains digital elevation model (DEM) data, typically used to model terrain on large, regular bodies such as the moon, Mars or Mercury.

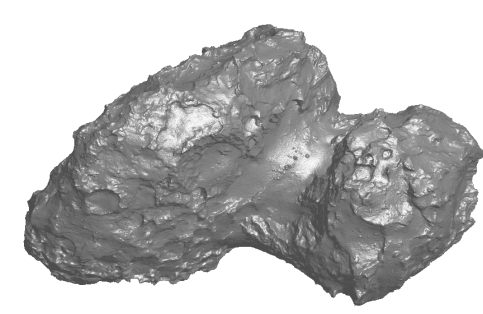

Figure 1 Rendering of comet Churyumov-Gershmenko based on a 4 million plate Digital Shape Kernel

As for the other SPICE subsystems, the data contained in a DSK is produced by others-SPICE just provides a container with allied computational capabilities allowing the user to compute observation geometry parameters such as location of the terminator, spacecraft altitude above the surface, or the location and lighting angles where an instrument's optical axis intercepts a body's surface.

Many means for handling shape data exist in the community. So why should NAIF jump on this bandwagon? The primary reason is to allow people using SPICE for many geometric computations to also use SPICE for computations involving all popular body shape data sets-not just tri-axial models. Additionally, NAIF believes the DSK approach offers extra features not found in some other shape model systems, such as the ability to access regions of shape data of differing resolution, and the ability to handle very large shape data sets that span multiple files, all within one program run.

A tutorial on the Digital Shape Kernel subsystem is available here:

http://naif.jpl.nasa.gov/pub/naif/toolkit_docs/Tutorials/pdf/indiv idual_docs/37_dsk.pdf.

2.2.3 Toolkit Performance Improvements Achieving high speed performance was not among the original SPICE Toolkit design considerations, but customers have often suggested NAIF try to improve speed to better meet their pipeline processing needs. NAIF has found several areas where lowlevel changes to Toolkit code has provided substantial improvement in computation speed while not violating NAIF's "no changes" policy. ("No changes" applies to the user's experience.) User's may see anywhere from no to perhaps $50 \%$ improvement in their SPICE computations depending on the kinds of computations being used.

\subsection{New SPICE Interfaces}

Since the inception of the system 26 years ago, a SPICE user has had to incorporate a handful of Toolkit APIs into his/her own application program to make needed geometry computations. That usage model remains the primary one today, but two new means of using SPICE have recently been made available to the space science community. 
2.3.1 WebGeocalc Tool Scientists and engineers often wish to make some sort of mission geometry computation without having to spend the time, or to have the know-how, to write a SPICE API-based program. NAIF has built a SPICE "geometry engine" incorporated in a client-server WWW-based architecture to address this need (Fig.2).

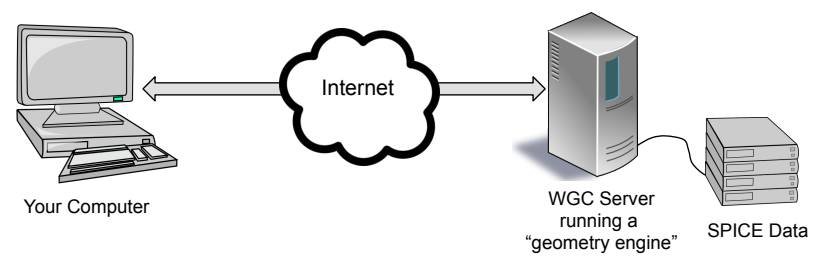

Figure 2 Architecture of the WebGeocalc Tool

A WebGeocalc (WGC) user needs only a computer with a standard web browser to make use of this tool. After connecting to the WGC server, a user makes use of standard Graphical User Interface (GUI) widgets to select the SPICE data to be used, the computation wanted, and the various parameters and options to be used in the computation such as time, target and observer objects, and reference frames and coordinate systems. Often the user may also specify plots to be made of WGC's numeric results. Pressing the "CALCULATE" button causes the WGC server to make the specified computation and return the numeric and any plotted results to the user's browser. The numeric results may be downloaded for use elsewhere, or may be saved for use as inputs in a subsequent WGC calculation-a process called chaining. Plots may also be downloaded for use in presentations.

NAIF's WebGeocalc server located at JPL has access to all of the planetary mission SPICE archives hosted there, plus a large set of generic (mission independent) SPICE data, and all of the current mission operations data for JPL-operated missions that use the SPICE system. It may be possible for other major planetary exploration enterprises to install a local WGC server tuned to their own needs.

A tutorial on WebGeocalc and the link to the tool are available here: http://naif.jpl.nasa.gov/naif/webgeocalc.html .

2.3.2 SPICE-Enhanced Cosmographia 3D Mission Visualization Tool Until recently the only form of SPICE output was numeric data. The advent of the WebGeocalc tool mentioned earlier allows for some graphical output. But NAIF users began asking if there was not some sort of readily available trajectory visualization tool that would use SPICE data as its primary source of information.

NAIF was aware of a number of SPICE-aware trajectory visualization tools, and even published pointers to these and other SPICE-aware tools. But it seemed those extant visualization tools did not offer all the characteristics NAIF felt would be important to the SPICE user community: fully SPICE based, portable to popular computing platforms, easy to use, unrestricted by license or export controls, and free of cost.

Serendipity stepped up in the form of an open source tool named Cosmographia, built in the U.S. by Fifth Star Labs. Some engineers at JPL had begun using this tool for future mission studies and asked for some NAIF help since the tool could use some kinds of SPICE data. NAIF staff liked what we saw and made arrangements with the Cosmographia author to add additional SPICE functionality and other features useful to the professional space science community.
This tool can display the orbits of spacecraft and natural bodies provided in SPICE ephemeris format. Bodies are rendered properly and with texture maps. A variety of vectors can be turned on, as can spacecraft and body reference frames (coordinate systems), Fig. 3. The "camera"-the user's viewpoint-can be changed in many ways. Instrument view cones can be displayed, and instrument footprints corresponding to actual (or planned) observations can be seen.

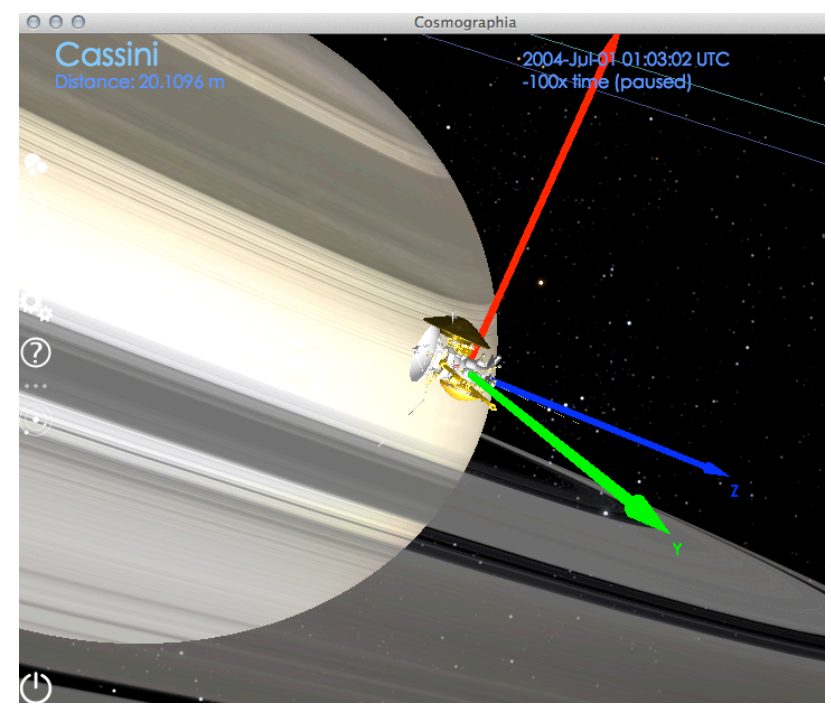

Figure 3 A snapshot taken from SPICE-Enhanced Cosmographia, showing the Cassini spacecraft near Saturn

Rather than try to list here all of its many features, the reader is referred to the SPICE-enhanced Cosmographia on-line User's Guide, found here: http://cosmoguide.org/.

More information and download instructions may be found here: http://naif.jpl.nasa.gov/naif/cosmographia.html.

\section{ACKNOWLEDGEMENTS}

The research described in this publication was carried out at the Jet Propulsion Laboratory, California Institute of Technology, under a contract with the National Aeronautics and Space Administration.

The starting point Cosmographia software was provided by Chris Laurel of Fifth Star Labs, and its rendering engine is provided by Astos Solutions GmbH. Eric Ferguson of JPL was the primary author of the enhancements to Cosmographia, in association with NAIF.

Mark Rose of NASA/Ames was the primary developer of the WebGeocalc tool, under direction of NAIF.

Copyright 2016 California Institute of Technology. U.S. Government sponsorship acknowledged.

\section{REFERENCES}

Acton, C.H.; "Ancillary Data Services of NASA's Navigation and Ancillary Information Facility;" Planetary and Space Science, Vol. 44, No. 1, pp. 65-70, 1996. 